\title{
Cysto-duodenal fistula: an unusual complication of a nonparasitic hepatic cyst
}
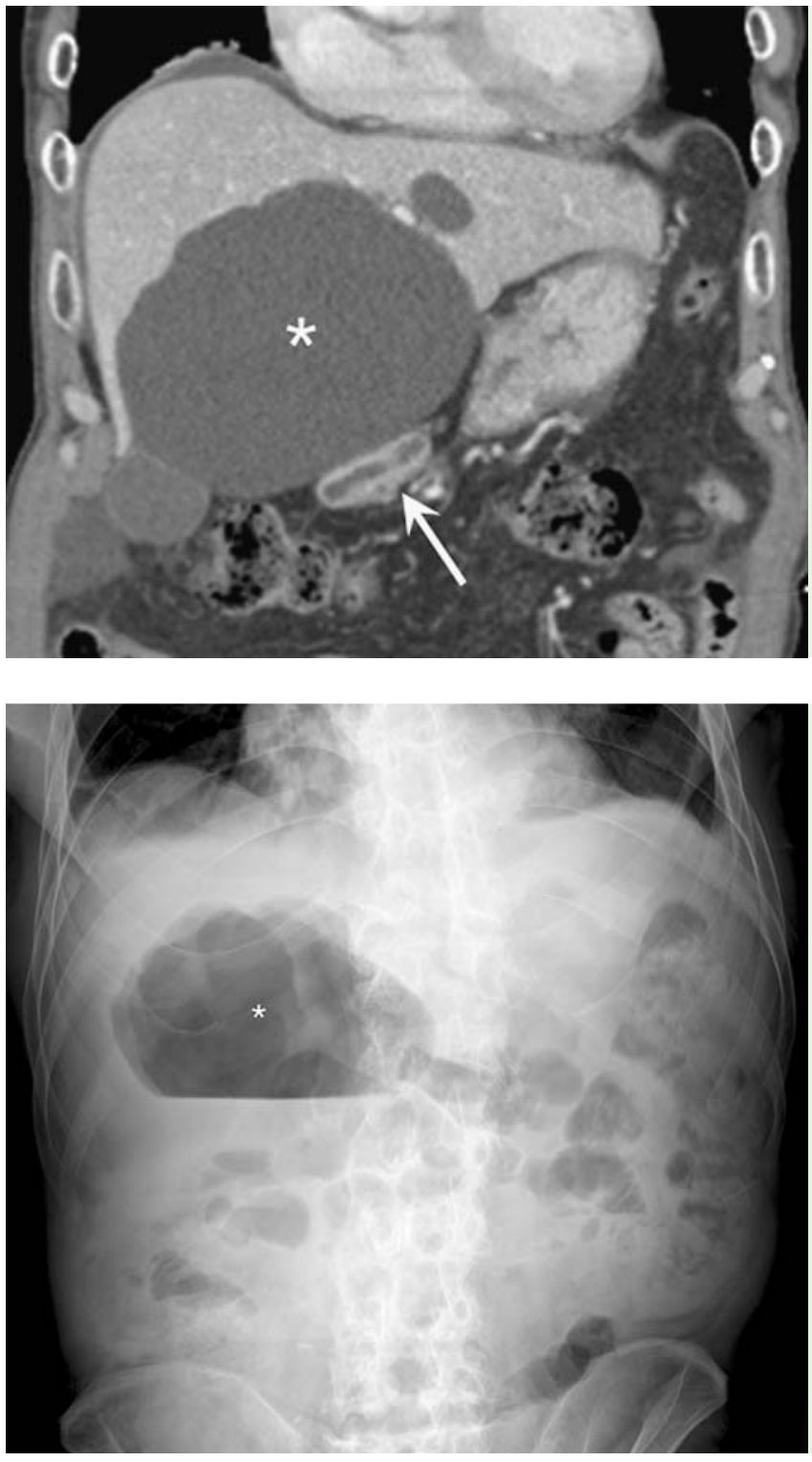

Increasing use of radiological imaging modalities has resulted in the detection of nonparasitic hepatic cysts in $1 \%-5 \%$ of the general population, however, complicated nonparasitic hepatic cysts are exceedingly rare [1-4]. Here, we present a unique case of a hepatic cysto-duodenal fistula with the intracystic endoscopic findings.

A 70-year-old man was admitted for postprandial abdominal discomfort and anemia of recent onset. Eight months previously, a $9 \times 12-\mathrm{cm}$ asymptomatic hepatic cyst in segments IV and V, which was compressing the duodenum, had been incidentally identified on computed tomog-
Fig. 1 Contrastenhanced computed tomography in a 70-year-old man with postprandial abdominal discomfort and anemia of recent onset. There is a huge homogeneous cyst (asterisk) in segments IV and $V$ of the liver, compressing the adjacent first portion of the duodenum (arrow).

Fig. 2 Simple abdominal radiographic view showing a large amount of intra-abdominal air (asterisk) with an internal air-fluid level in the right upper quadrant of the abdomen.

raphy (CT) ( Fig.1). On admission, simple abdominal radiography showed welldelineated, intra-abdominal gas with an internal air-fluid level in the right upper quadrant of the abdomen, but no intraabdominal free air ( Fig.2). Laboratory studies revealed anemia (hemoglobin $7.5 \mathrm{~g} / \mathrm{dL}$, hematocrit $23.7 \%$, and serum ferritin $286.1 \mathrm{ng} / \mathrm{mL}$ ) compatible with acute blood loss.

Esophagogastroduodenoscopy, using a conventional gastroscope, showed a 10$\mathrm{mm}$ penetrated peptic ulcer at the anterior wall of the duodenal bulb ( Fig.3). An ultrathin endoscope (GIFXP260N, Olympus Optical Co., Tokyo,

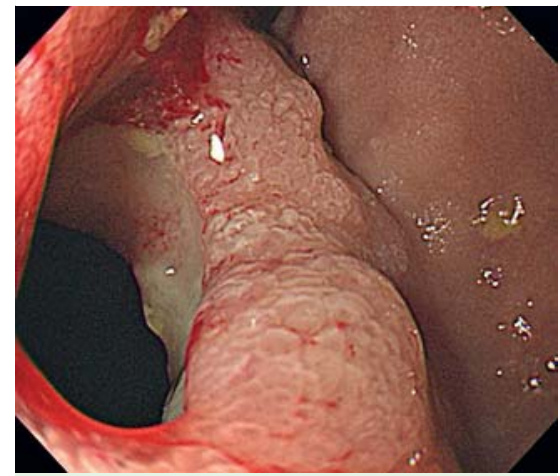

Fig. 3 Endoscopic view showing a 10-mm penetrated peptic ulcer at the anterior wall of the duodenal bulb.
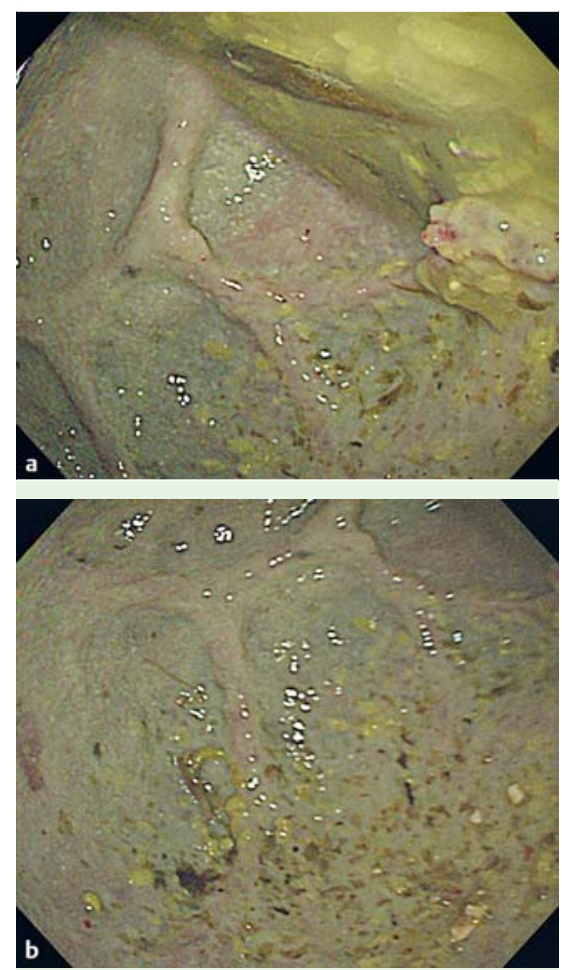

Fig. 4 a, b Intracystic endoscopic findings using an ultrathin endoscope: note the thick feeding vessels and food materials in the cavity wall.

Japan), passed through the opening, revealed a huge unilocular cystic cavity with thick feeding vessels and food materials in the cyst wall ( Fig.4). However, there was no evidence of intracystic hemorrhage or abscess formation. Abdominal CT verified internal communication between the pre-existing hepatic cyst and the duodenal bulb through a fistulous 
tract. Considering the underlying mechanism of fistula formation, we speculated that persistent compression and subsequent mechanical friction due to the presence of the cyst resulted in progressive erosion of the adjacent duodenal wall. The spontaneous development of a pepticduodenal ulcer might have played a role as well. The patient was treated with a proton-pump inhibitor and he remained healthy during the 8-month followup, with gradual improvement of anemia. He refused further followup examinations.

Endoscopy_UCTN_Code_CCL_1AB_2AG_3AC

Competing interests: None
Jung W. Kim, Chang K. Lee, Jae J. Shim, Jae Y. Jang

Division of Gastroenterology, Department of Internal Medicine, Kyung Hee University School of Medicine, Seoul, Korea

\section{References}

1 Lai EC, Wong J. Symptomatic nonparasitic cysts of the liver. World J Surg 1990; 14 : $452-456$

2 Regev A, Reddy KR, Berho $M$ et al. Large cystic lesions of the liver in adults: a 15-year experience in a tertiary center. J Am Coll Surg 2001; 193: 36-45

3 Ishikawa H, Uchida S, Yokokura Y et al. Nonparasitic solitary huge liver cysts causing intracystic hemorrhage or obstructive jaundice. J Hepatobiliary Pancreat Surg 2002; 9: $764-768$

4 Garcea G, Pattenden CJ, Stephenson J et al. Nine-year single-center experience with nonparastic liver cysts: diagnosis and management. Dig Dis Sci 2007; 52: 185-191
Bibliography

DOI http://dx.doi.org/ 10.1055/s-0033-1344827

Endoscopy 2013; 45: E382-E383

(c) Georg Thieme Verlag KG

Stuttgart · New York

ISSN 0013-726X

\section{Corresponding author}

\section{Chang K. Lee}

Department of Internal Medicine Kyung Hee University School of Medicine 23 Kyungheedae-ro

Dongdaemun-gu

Seoul 130-872

Korea

Fax: +82-2-9681848

cklee92@paran.com 